\title{
Understanding the WMAP Results: Low-Order Multipoles and Dust in the Vicinity of the Solar System
}

\author{
Valeri Dikarev · Oliver Preuß · Sami Solanki · Harald Krüger · \\ Alexander Krivov
}

Received: 1 July 2007 / Accepted: 4 October 2007 / Published online: 26 October 2007

(C) Springer Science+Business Media B.V. 2007

\begin{abstract}
Analyses of the cosmic microwave background (CMB) radiation maps produced by the Wilkinson Microwave Anisotropy Probe (WMAP) have revealed anomalies not predicted by the standard cosmological theory. It has been suggested that a dust cloud in the vicinity of the Solar system may be the cause for these anomalies. In this paper, the thermal emission by particles from two known interplanetary meteoroid complexes is tested against the CMB maps. Conclusions are drawn based on the geometry of cloud projections onto the WMAP sky whether these clouds are likely to explain the observed anomaly. The smooth background Zodiacal cloud and one of the Taurid meteor complex branches do not explain the WMAP anomaly.
\end{abstract}

Keywords CMB $\cdot$ WMAP $\cdot$ Solar system $\cdot$ Dust

\section{Introduction}

The cosmic microwave background $(\mathrm{CMB})$ radiation was released $14 \times 10^{9}$ years ago, 380,000 years after the Big Bang, i.e. when the Universe became transparent. Ever cooling since then, the CMB has reached the average temperature of $2.725 \mathrm{~K}$ today. Fluctuations of the $\mathrm{CMB}$ around this temperature, of the order of $100 \mu \mathrm{K}$, carry information about matter and gravitational potential distribution at the earliest time of the Universe. Thus cosmological theories can be tested against maps of CMB.

V. Dikarev $(\bowtie) \cdot$ O. Preuß $\cdot$ S. Solanki $\cdot$ H. Krüger

MPI für Sonnensystemforschung, 37191 Katlenburg-Lindau, Germany

e-mail: dikarev@mps.mpg.de

V. Dikarev

MPI für Kernphysik, 69117 Heidelberg, Germany

V. Dikarev

Astronomical Institute of St. Petersburg University, St. Petersburg, Russia

A. Krivov

AIU FSU Jena, Jena, Germany 
The Wilkinson Microwave Anisotropy Probe (WMAP, see Bennett et al. 2003) has surveyed the microwave sky at a so far unprecedented accuracy and resolution, allowing one to make maps of CMB fluctuations and analyse them (Bennet et al. 2003; Hinshaw et al. 2007). As the fluctuations are random, and the sky is a single implementation, statistical properties of the fluctuations are determined. The entire map is expanded into spherical harmonic functions, and cosmological theories are applied to predict the power of the multipoles obtained from the data. While the standard inflationary cosmology is confirmed by the WMAP data at a very high confidence level for nearly all multipole orders, significant contradictions have been found as well. In particular, contrary to predicted independence, the quadrupole and octopole moments are mutually aligned to a very unlikely degree. Moreover, they are aligned with the Solar-system geometry at a confidence level that is again difficult to ignore (see Fig. 1).

It has been suggested that a dust cloud in the vicinity of the Solar system could be the reason for the anomaly (Copi et al. 2006; de Oliveira-Costa and Tegmark 2006; Schwarz et al. 2004; Starkman and Schwarz 2005). Calculations of the thermal emission spectra of interplanetary meteoroids performed in Dikarev et al. (2007) have shown that mm-sized carbonaceous as well as $\mathrm{cm}$-sized silicate particles are characterised by a flat absorption efficiency throughout the infrared and millimetre wavelengths, whereas the absorption efficiency of small dust grains of a few tens micrometres in size and below, decreases steeply with the wavelength $\lambda$ increase, close to the emissivity law $\lambda^{-2}$ usually applied to describe spectra of fine grains. In order to provide the microwave emission sufficient to cause the
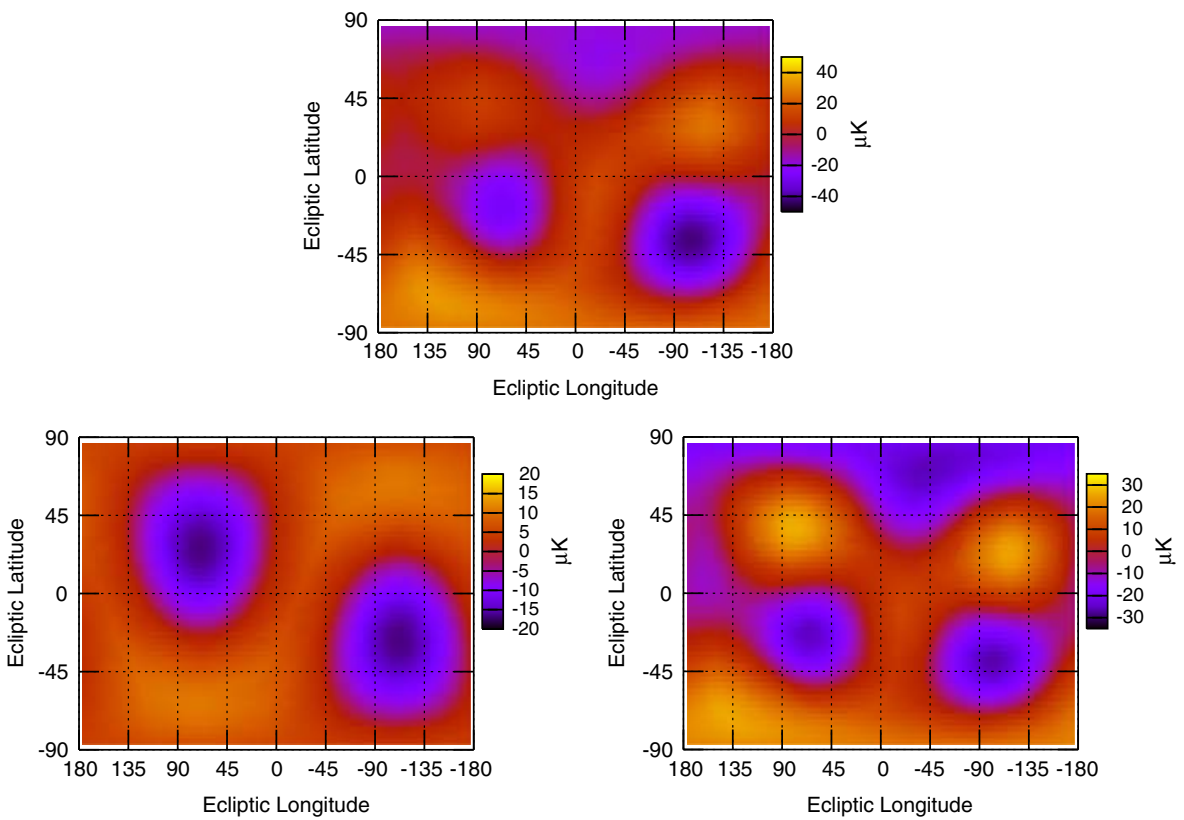

Fig. 1 Quadrupole (bottom left) and octopole (bottom right) from the three-year temperature map (Hinshaw et al. 2007), and their superposition (top) in the ecliptic coordinate system. A first look at the octopole component suggests an unusual planarity compared to what is expected from an isotropic random field with no preferred axis. Also the quadrupole and octopole show a striking correlation with the ecliptic plane which clearly separates three extrema in the north and three in the south. In addition, the superposition map reveals a north-south ecliptic asymmetry where the extrema in the north are visibly weaker than those in the south 
WMAP anomaly, one would therefore need a cloud of big particles with a significantly smaller visual and infrared optical depth than under the hypothesis of a cloud of tiny grains, possibly explaining why this cloud has remained unknown. The large meteoroids, however, have been observed only scarcely, almost entirely as meteors in Earth's atmosphere. Their number densities in the Solar system remain largely unconstrained. Therefore, we constructed relative microwave brightness maps of several known dust clouds, assuming that the big particles may be more abundant in these clouds than it was previously thought. In this paper, we report two of this series of tests of existing dust clouds against the WMAP data that we have carried out in order to find the mysterious cause of the WMAP anomaly.

\section{Projecting Dust Clouds onto the WMAP Sky}

The full sky maps made by the WMAP are dominated by the CMB fluctuations, which are Gaussian random, and appear to be too noisy to put any firm constraints on the dust cloud that may be responsible for the quadrupole and octopole anomalies. Therefore, instead of a comparison between the full maps and dust cloud images, we make a comparison of the quadrupole and octopole moments of CMB fluctuations, i.e. where the hypothetical dust cloud is best revealed, and the corresponding momenta of the multipole expansions of real dust cloud images.

The expansion is implemented as an integration over the entire celestial sphere:

$$
a_{l, n}=\int Y_{l, n}^{*}(\Omega) f(\Omega) \mathrm{d} \Omega,
$$

where $Y_{l, n}$ is a spherical harmonic function and $a_{l, n}$ is the corresponding expansion coefficient, $f$ is the function expanded, $l=1, \ldots, \infty$, and $n=-l, \ldots, l$. The quadrupole and octopole momenta are indexed by $l=2$ and 3 , respectively.

The expanded function $f$ is defined so as to simulate the dust cloud number density, temperature and thermal emission, and the WMAP observation strategy. For each line of sight, the integral

$$
I=\int_{0}^{\infty} B[\lambda, T(r)] C_{\mathrm{abs}}[x(l), y(l), z(l)] \mathrm{d} l
$$

is evaluated, where $B$ is the blackbody emission power at the wavelength $\lambda, C_{\mathrm{abs}}$ is the absorption cross-section of dust per unit volume, assumed to be proportional to the number density of dust, $l$ is the position on the line of sight, $x, y$, and $z$ are its heliocentric coordinates. The temperature $T$ of dust is adopted from the model (Kelsall et al. 1998)

$$
T(r)=286 \mathrm{~K} \times(1 \mathrm{AU} / r)^{0.467} .
$$

The WMAP observation strategy is approximately described as viewing from the moving Earth everywhere within the range of solar elongations from $90^{\circ}$ to $135^{\circ}$, with a uniform coverage inside this range (c.f. Bennet et al. 2003).

\section{Dusty Multipoles}

The Zodiacal cloud is a flat cloud nearly symmetric about a plane slightly inclined with respect to the ecliptic plane. If its inclination were zero, one would not expect it to cause 
the anomalies reported by the WMAP. However, the inclination is about $2^{\circ}$, and the half latitudinal width at half maximum of the number density is about $15^{\circ}$. Therefore the motion of the Earth (and WMAP) with respect to the cloud's symmetry plane causes e.g. polar brightness variations of 20 to $30 \%$ due to the column density variations (Kelsall et al. 1998)! Also, the Earth's heliocentric distance oscillations lead to the temperature variations of dust on the same rest-frame line of sight. It seems to be worthwhile to check if the motion of the WMAP observatory with respect to this cloud can explain the anomalous multipoles.

The major component of the model (Kelsall et al. 1998), the smooth background cloud, is implemented. Even though the relevance of that model fitted to the infrared data to the interplanetary dust seen in the microwaves is doubtful, there is an argument in support of its use in rough first-step estimates. To put it simply, the model is not worse than any other number density representation in resembling the most general, large-scale properties of the zodiacal dust cloud, i.e. a flat, azimuthally symmetric dust complex inclined with respect to the ecliptic plane.

The smooth background cloud emission maps are plotted in Fig. 2. The top row consists of two maps of the total emission, before the expansion into multipoles, in arbitrary units, at $1 \mathrm{~mm}$ (left) and $5 \mathrm{~mm}$ (right) wavelengths. Substantially different in normalisations, their relative distributions are also different, since at $5 \mathrm{~mm}$ more remote and colder dust is seen, which extends further in the ecliptic plane, while the number density drops down exponentially in the vertical direction.

To some extent in accord with expectations, the octopole moment of the smooth background cloud does reveal an interesting structure with maxima and minima following anti-symmetrically along the ecliptic longitude. The quadrupole, however, is closely confined to the ecliptic plane. Moreover, the octopole magnitude with respect to that of the quadrupole is negligible: less than $1 \%$, contrary to the WMAP results which suggest similar quadrupole and octopole magnitudes.

In a 'gedanken experiment', we tried to enhance the anticipated effects of the cloud's inclination and Earth's orbital eccentricity by artificially increasing both of them. This leads indeed to more equal quadrupole and octopole magnitudes, with a structure reminiscent of the WMAP multipoles, although not identical. As neither cloud's inclination nor Earth's eccentricity can be modified, the next idea is to find an existing meteoroid complex in the Solar system that is on an eccentric and inclined orbit.

One possible candidate for such a complex is the Taurid meteor complex. It was interesting to simulate as well, since some believed (Štohl and Porubčan 1991; Whipple and Hamid 1952) it is related to comet 2P/Encke and may have been produced in a recent disruption of a large comet, perhaps by collision with an asteroid. This can make the complex potentially a large modern reservoir of big meteoroids being collisionally transformed into small dust.

The orbit elements of one of the Taurid's radiants are adopted, the semimajor axis 2.2 AU, eccentricity 0.8 , inclination $3.6^{\circ}$, perihelion arguments $294^{\circ}$, and node longitude $225^{\circ}$ (Štohl and Porubčan 1991). Figure 3 shows how the Taurid meteor stream would look as viewed from the Sun. The longitude of pericentre is indeed near $180^{\circ}$ ecliptic longitude where the figure shows an enlargement of the stream thickness due to its proximity, while the surface brightness peaks near zero longitude where cold dust dwells near the aphelion. Figure 4 shows the emission from the Taurid meteor complex as seen by WMAP. The bizarre appearance of the stream in the top left panel of Fig. 4 is due to the motion of the Earth and WMAP observation strategy. 

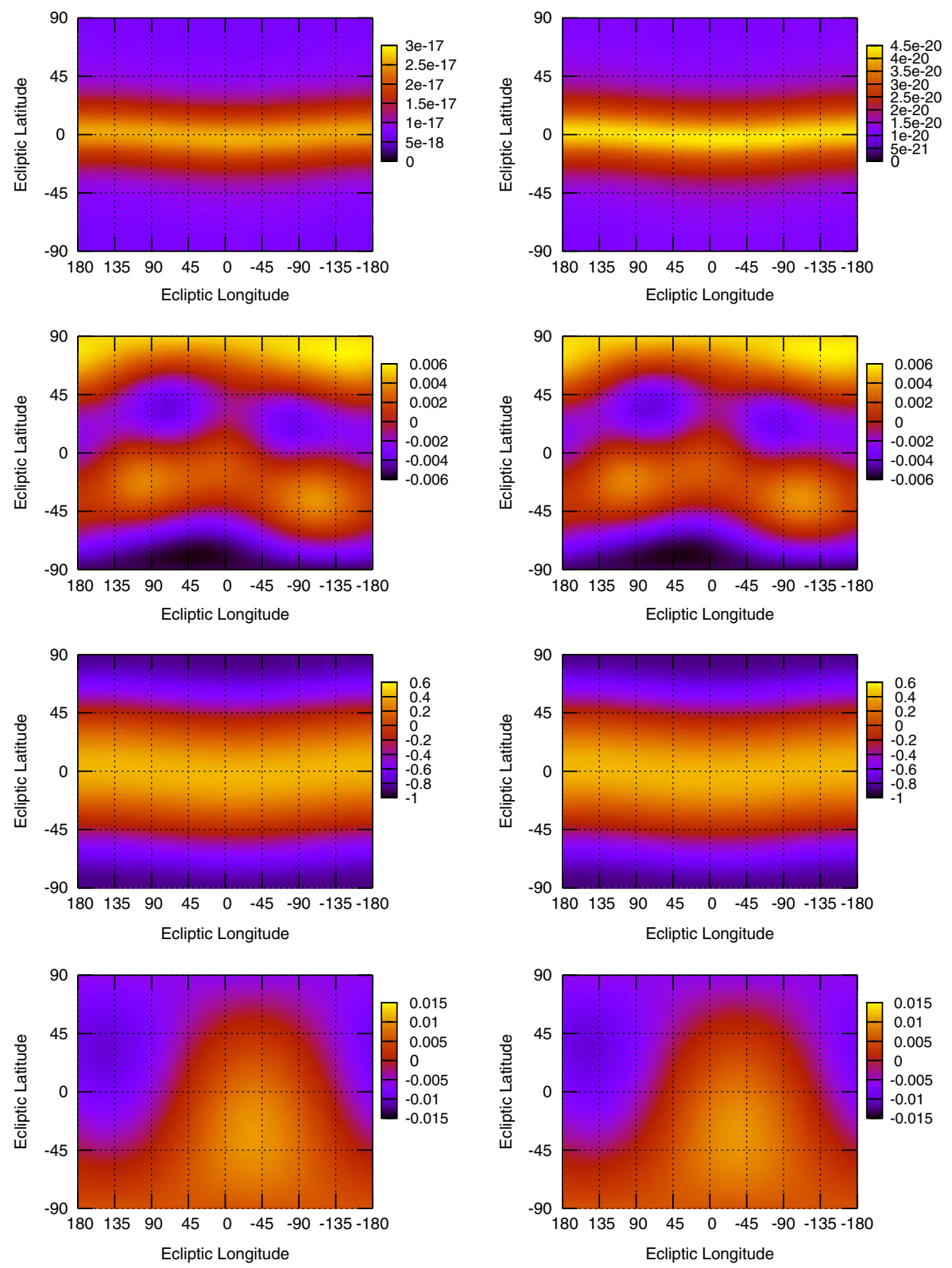

Fig. 2 Maps of the dust emission due to the smooth background zodiacal cloud of Kelsall et al. (1998). Left column: $1 \mathrm{~mm}$ wavelength, right column: $5 \mathrm{~mm}$. From top to bottom: the total emission in arbitrary units, then the octopole, quadrupole and dipole in units of the monopole

Interestingly, one can see indeed comparable magnitudes of the quadrupole and octopole, probably because all the low-order multipoles are equally bad at reproducing the lowscale features. Yet their alignment is not obviously close to the WMAP puzzling results. Moreover, as most of the multipole power comes from a very small spot on the sky, and if 
Fig. 3 Maps of the thermal emission by meteoroids of the Taurid meteor complex at the wavelength of $1 \mathrm{~mm}$, as it would appear to an observer at the Sun, arbitrary units
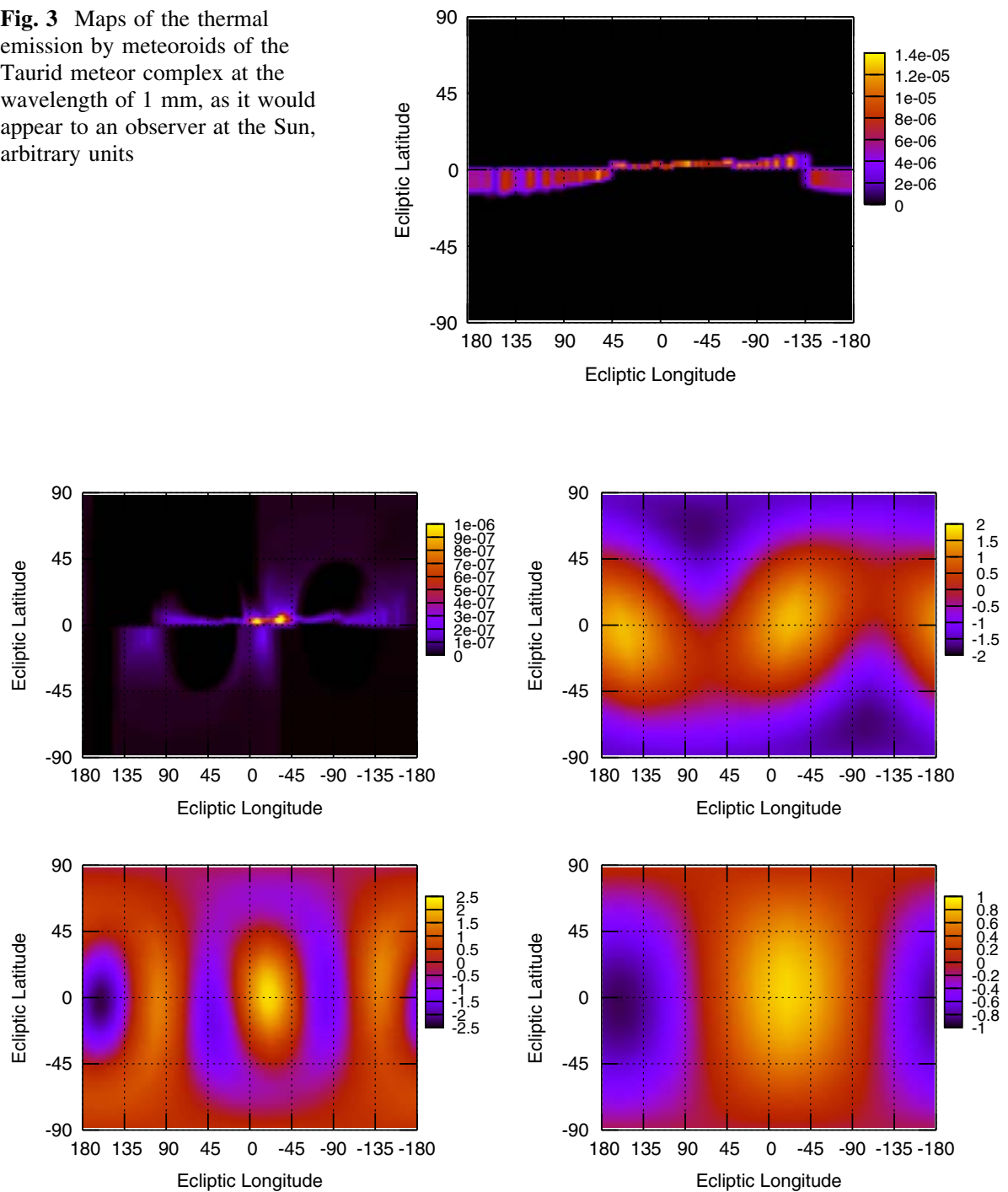

Fig. 4 Maps of the thermal emission by the meteoroids of the Taurid meteor complex at the wavelength of $1 \mathrm{~mm}$. Top left: the total emission, before the expansion into multipoles, in arbitrary units; bottom left: the octopole; top right: the quadrupole; bottom right: the dipole; the multipoles are in the units of monopole

indeed this single bright spot were responsible for the alignment of low- $l$ multipoles, then it would certainly be recognized on the full sky maps, even before the multipole expansion procedure was applied. This is not the case, however, so this hypothesis can also be rejected.

\section{Conclusion}

We have reported an ongoing search for the reason of the WMAP anomaly. An unexpected alignment of the quadrupole and octopole moments of the expansion of the map of CMB 
fluctuations mutually and with the Solar-system geometry was interpreted as an indication of the presence of a dust cloud that radiates in the microwaves. In this paper, the smooth background component of the Zodiacal cloud and the Taurid meteor complex are both tested for compatibility with the CMB quadrupole and octopole. They are both rejected based on different argumentation. We plan to consider more known and hypothetical dust clouds, bound to and neighbouring the Solar system, in our subsequent publications (Dikarev et al. 2007).

\section{References}

C.L. Bennett, M. Bay, M. Halpern, G. Hinshaw, C. Jackson, N. Jarosik, A. Kogut, M. Limon, S.S. Meyer, L. Page, D.N. Spergel, G.S. Tucker, D.T. Wilkinson, E. Wollack, E.L. Wright, The microwave anisotropy probe mission. Astrophys. J. 583, 1-23 (2003)

C.L. Bennett, M. Halpern, G. Hinshaw, N. Jarosik, A. Kogut, M. Limon, S.S. Meyer, L. Page, D.N. Spergel, G.S. Tucker, E. Wollack, E.L. Wright, C. Barnes, M.R. Greason, R.S. Hill, E. Komatsu, M.R. Nolta, N. Odegard, H.V. Peiris, L. Verde, J.L. Weiland, First-year Wilkinson microwave anisotropy probe (WMAP) observations: preliminary maps and basic results. Astrophys. J. Suppl. 148, 1-27 (2003). doi: 10.1086/377253, arXiv:astro-ph/0302207

C.J. Copi, D. Huterer, D.J. Schwarz, G.D. Starkman, On the large-angle anomalies of the microwave sky. Mon. Not. R. Astron. Soc. 367, 79-102 (2006). doi: 10.1111/j.1365-2966.2005.09980.x, arXiv:astro-ph/ 0508047

A. de Oliveira-Costa, M. Tegmark, CMB multipole measurements in the presence of foregrounds. Phys. Rev. D 74(2) (2006), 023,005-+, arXiv:astro-ph/0603369

V. Dikarev, O. Preuß, S. Solanki, H. Krüger, A. Krivov, The local dust foregrounds in the microwave sky: I. Thermal emission spectra. Astrophys. J. submitted (2007)

G. Hinshaw, M.R. Nolta, C.L. Bennett, R. Bean, O. Doré, M.R. Greason, M. Halpern, R.S. Hill, N. Jarosik, A. Kogut, E. Komatsu, M. Limon, N. Odegard, S.S. Meyer, L. Page, H.V. Peiris, D.N. Spergel, G.S. Tucker, L. Verde, J.L. Weiland, E. Wollack, E.L. Wright, Three-year Wilkinson microwave anisotropy probe (WMAP) observations: temperature analysis. Astrophys. J. Suppl. 170, 288-334 (2007). doi: 10.1086/513698, arXiv:astro-ph/0603451

T. Kelsall, J.L. Weiland, B.A. Franz, W.T. Reach, R.G. Arendt, E. Dwek, H.T. Freudenreich, M.G. Hauser, S.H. Moseley, N.P. Odegard, R.F. Silverberg, E.L. Wright, The COBE diffuse infrared background experiment search for the cosmic infrared background. II. Model of the interplanetary dust cloud. Astrophys. J. 508, 44-73 (1998)

D.J. Schwarz, G.D. Starkman, D. Huterer, C.J. Copi, Is the low- $\ell$ microwave background cosmic? Phys. Rev. Lett. 93(22) (2004), 221,301-+. doi: 10.1103/PhysRevLett.93.221301, astro-ph/0403353

G.D. Starkman, D.J. Schwarz, Is the universe out of tune? Sci. Am. 291, 36-43 (2005)

J. Štohl, V. Porubčan, Dinamical aspects of the Taurid meteor complex, in Chaos, Resonance and Collective Dynamical Phenomena in the Solar System, ed. by S. Ferraz-Mello (1991), pp. 315-324

F.L. Whipple, S.E. Hamid, On the origin of the Taurid meteor streams, in Helman Obs. Bull. 41, Harward Reprint 361 (1952), pp. 1-30 